\title{
Subgenual Cingulate-Amygdala Functional Disconnection and Vulnerability to Melancholic Depression
}

\author{
Clifford I Workman ${ }^{1,2}$, Karen E Lythe'2, Shane McKie', Jorge Moll', Jennifer A Gethin', John FW Deakin', \\ Rebecca Elliott' and Roland Zahn*,2,4
}

'The University of Manchester and Manchester Academic Health Sciences Centre, Institute of Brain, Behaviour and Mental Health, Neuroscience and Psychiatry Unit, Manchester, UK; ${ }^{2}$ The University of Manchester and Manchester Academic Health Sciences Centre, School of Psychological Sciences, Neuroscience and Aphasia Research Unit, Manchester, UK; ${ }^{3}$ Cognitive and Behavioral Neuroscience Unit, D'Or Institute for Research and Education (IDOR), Rio de Janeiro, Brazil; ${ }^{4}$ Institute of Psychiatry, Psychology, and Neuroscience, Department of Psychological Medicine, Centre for Affective Disorders, King's College London, London, UK

\begin{abstract}
The syndromic heterogeneity of major depressive disorder (MDD) hinders understanding of the etiology of predisposing vulnerability traits and underscores the importance of identifying neurobiologically valid phenotypes. Distinctive fMRI biomarkers of vulnerability to MDD subtypes are currently lacking. This study investigated whether remitted melancholic MDD patients, who are at an elevated lifetime risk for depressive episodes, demonstrate distinctive patterns of resting-state connectivity with the subgenual cingulate cortex (SCC), known to be of core pathophysiological importance for severe and familial forms of MDD. We hypothesized that patterns of disrupted SCC connectivity would be a distinguishing feature of melancholia. A total of 63 medication-free remitted MDD (rMDD) patients (33 melancholic and 30 nonmelancholic) and 39 never-depressed healthy controls (HC) underwent resting-state fMRI scanning. SCC connectivity was investigated with closely connected bilateral a priori regions of interest (ROls) relevant to MDD (anterior temporal, ventromedial prefrontal, dorsomedial prefrontal cortices, amygdala, hippocampus, septal region, and hypothalamus). Decreased (less positive) SCC connectivity with the right parahippocampal gyrus and left amygdala distinguished melancholic rMDD patients from the nonmelancholic rMDD and HC groups (cluster-based familywise error-corrected $p \leqslant 0.007$ over individual a priori ROls corresponding to approximate Bonferroni-corrected $p \leqslant 0.05$ across all seven a priori ROls). No areas demonstrating increased (more positive) connectivity were observed. Abnormally decreased connectivity of the SCC with the amygdala and parahippocampal gyrus distinguished melancholic from nonmelancholic rMDD. These results provide the first resting-state neural signature distinctive of melancholic rMDD and may reflect a subtype-specific primary vulnerability factor given a lack of association with the number of previous episodes. Neuropsychopharmacology (2016) 4I, 2082-2090; doi:I 0.1038/npp.20I6.8; published online I0 February 2016
\end{abstract}

\section{INTRODUCTION}

Major depressive disorder (MDD) is an inherently heterogeneous condition because of its polythetic diagnostic criteria. A diagnosis of MDD requires a combination of only five of nine possible symptoms, meaning two patients may share only one common symptom. This heterogeneity decreases the likelihood of identifying the underlying neurobiological mechanisms underpinning MDD and underscores the importance of identifying neurobiologically valid depressive phenotypes. The melancholic subtype of MDD, characterized by unvarying low mood and drive as well as psychomotor and vegetative symptoms, has been

*Correspondence: Dr R Zahn, Institute of Psychiatry, Psychology, and Neuroscience, Department of Psychological Medicine, Centre for Affective Disorders, King's College London, London SE5 8AZ, UK, Tel: +44 (0)20 7848 0348, Fax: +44 (0)20 7848 0298,

E-mail: roland.zahn@kcl.ac.uk

Received 25 August 2015; revised 6 January 2016; accepted 12 January 2016; accepted article preview online 19 January 2016 associated with stable personality features that are present outside the depressed state and increase vulnerability to depression (Hecht et al, 1998). Identifying a distinct neural signature for vulnerability to melancholic MDD would provide important evidence of its neurobiological validity and point to an intermediate phenotype (Meyer-Lindenberg and Weinberger, 2006). Neuroimaging studies in patients with current melancholic MDD have provided initial evidence of distinct brain abnormalities including subgenual cingulate cortex (SCC) dysfunction (Pizzagalli et al, 2004). However, attempts at identifying markers capable of distinguishing melancholic MDD from other depressive subtypes have been largely unsuccessful. It is furthermore unclear whether the distinctive abnormalities described were correlates of the depressed state rather than vulnerability traits.

Resting-state functional MRI (rsfMRI), which can be used to measure spontaneous low-frequency fluctuations in blood oxygen level-dependent (BOLD) signal in the resting brain (Fox and Raichle, 2007), has become an increasingly popular 
imaging method for characterizing network-level disruptions associated with psychiatric disorders. There are strong theoretical and practical motivations for acquiring rsfMRI in psychiatric studies: (1) large metabolic demands of the resting brain suggest a critical role in overall brain functioning (Fox and Raichle, 2007), and (2) rsfMRI scans entail short acquisition times and do not require complex cognitive paradigms. The literature describing resting-state network abnormalities in current MDD has grown substantially over the past decade (reviewed in Dutta et al, 2014). Importantly, a recent rsfMRI study of patients with current MDD revealed decreased effective connectivity in attention and interoception networks in melancholic relative to nonmelancholic patients and a healthy control (HC) group (Hyett et al, 2015). It is elusive, however, whether these effects reflected differences in symptoms experienced during the depressive episode or neural differences irrespective of current symptom profile. It was further difficult to control for antidepressant effects. Therefore, it remains unknown whether rsfMRI can be used to reveal distinctive signatures of vulnerability traits predisposing to different MDD subtypes.

Studies of brain functioning in MDD highlight the SCC as a key region in the pathophysiology of depression (see Mayberg, 2003; Ressler and Mayberg, 2007). In HC participants, SCC BOLD response was associated with guilt proneness (Zahn et al, 2009) that is often excessive and overgeneralized in current MDD, particularly in the melancholic subtype. Resting-state glucose metabolism in the SCC has previously been shown to be elevated in current MDD and to normalize upon remission from the depressed state (reviewed in Ressler and Mayberg, 2007). Furthermore, current MDD patients demonstrated greater resting-state connectivity of the SCC with fronto-parieto-limbic regions relative to control participants (Greicius et al, 2007; Sheline et al, 2010). Green et al (2012) recently demonstrated taskrelated SCC decoupling in remitted MDD (rMDD) patients during the experience of guilt. As rMDD patients are at a highly elevated lifetime risk for major depressive episodes (MDEs) relative to HC participants (Kupfer, 1991), this suggests guilt-related SCC decoupling may represent a trait vulnerability factor for MDD rather than a correlate of the depressed state. In addition, although the literature on resting-state connectivity in depression vulnerability is limited, disrupted SCC connectivity has been observed in young rMDD patients (Gaffrey et al, 2012) and in at-risk adolescents (Herringa et al, 2013).

The present study employed rsfMRI to investigate functional connectivity of the SCC in fully remitted, medicationfree MDD patients with or without a history of melancholic MDEs. By investigating patients with $\mathrm{rMDD}$, the present study is well suited to identify stable vulnerability traits for experiencing MDEs (Burcusa and Iacono, 2007). The current study used a seed-based analysis approach as this method has been commonly used in rsfMRI studies of current MDD (Dutta et al, 2014). We used the SCC seed region identified in the Green et al (2012) study that previously demonstrated decoupling with medial frontal and medial and anterior temporal cortices during the experience of guilt in a rMDD patient group that included a high proportion of melancholic rMDD patients. We hypothesized that melancholic rMDD patients would demonstrate a distinctive pattern of disrupted
SCC connectivity when compared with the nonmelancholic rMDD patients and $\mathrm{HC}$ participants. We further predicted that decreased (less positive) medial prefrontal connectivity with the SCC would distinguish melancholic from nonmelancholic rMDD patients. This prediction was based on evidence that the medial prefrontal cortex is necessary for social actions and motivations (Moll et al, 2005) that are classically impaired in melancholia (Ebert et al, 1995), as well as its known direct anatomical connections to the SCC (Carmichael and Price, 1996; Vogt and Pandya, 1987) and previous work in which it demonstrated guilt-related functional disconnection with the SCC (Green et al, 2012).

\section{MATERIALS AND METHODS}

\section{Participants}

The present study was approved by the South Manchester National Health Service Research Ethics Committee. After the study procedures were explained in full, participants gave informed consent (verbal consent for a telephone prescreening and written consent at the outset of each study visit). Participant recruitment was conducted using online and print advertisements as part of the UK Medical Research Council-funded 'Development of Cognitive and Imaging Biomarkers Predicting Risk of Self-Blaming Bias and Recurrence in Major Depression' project (Zahn et al, 2015). Participants received compensation for their time and travel expenses. Before taking part in the study, 707 volunteers completed a telephone prescreening to preliminarily assess eligibility (the prescreening document is available at http://www.translational-cognitive-neuroscience.org/start/test-materials). Volunteers who passed the initial screening were then invited to complete a clinical interview overseen by a senior psychiatrist (RZ) at which psychiatric, clinical, and family histories were recorded. The Structured Clinical Interview-I for DSM-IV-TR was used to diagnose past MDEs with or without melancholic features (First et al, 2002) with moderate to perfect inter-rater reliability (see Supplementary Table S1). Exclusion criteria were: current axis I disorders, history of substance abuse or major medical or neurological disorders, exposure to psychotropic medications within 4 weeks (8 weeks for fluoxetine), and contraindications for MRI scanning. In addition, the HC group had no history of axis I disorders and no first-degree family history of mood disorders or schizophrenia. Following the initial clinical interview, 96 rMDD patients and $48 \mathrm{HC}$ participants were eligible for enrollment into the current study (see Table 1 for a detailed overview of reasons for exclusion). Of these, 63 medicationfree patients with rMDD (33 melancholic and 30 nonmelancholic) and 39 never-depressed HC participants underwent MRI scanning. One HC participant's data were excluded because of the presence of a pituitary abnormality.

The rMDD patients (both overall and melancholic) and HC group were closely matched on demographic variables (Table 2), as were the melancholic and nonmelancholic rMDD patients (Tables 2 and 3). The rMDD patients (both overall and melancholic) did not differ from HC group with respect to age, years of education, or sex. Scores on the Beck Depression Inventory (BDI; (Beck et al, 1996)) were slightly elevated in the rMDD patients and differed from the HC 
Table I Reasons for Exclusion of Volunteers from the Current Study

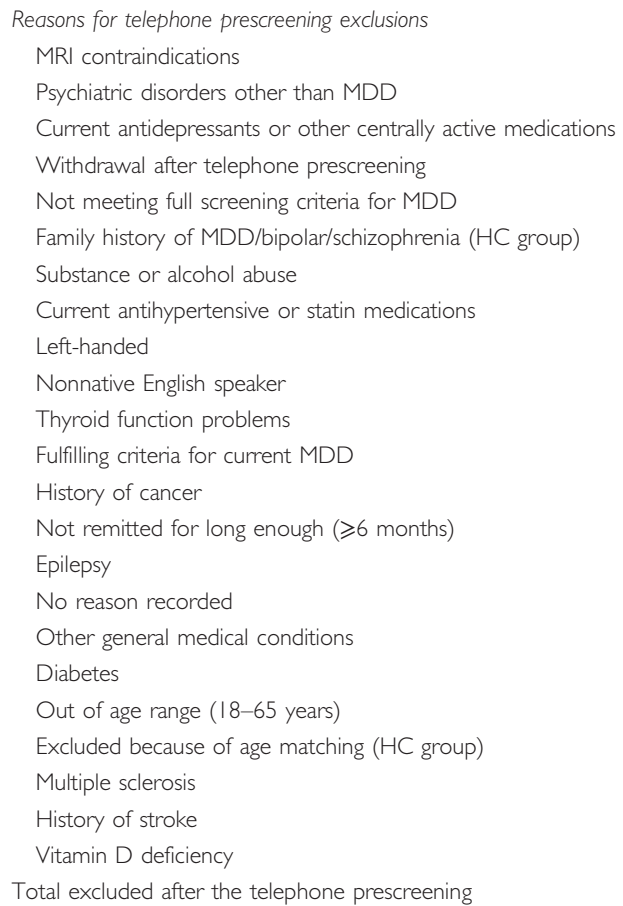

Abbreviations: $\mathrm{fMRl}$, functional magnetic resonance imaging; $\mathrm{HC}$, healthy control; MDD, major depressive disorder, MDE, major depressive episode.

Of the 707 volunteers who completed the telephone prescreening, 276 were eligible ( 84 remitted MDD patients and $92 \mathrm{HC}$ participants). Of these, 202 participants agreed to complete the clinical interview after having reviewed the study's participant information sheet (I 38 remitted MDD patients and $64 \mathrm{HC}$ participants). Following the clinical interview, 144 participants were eligible to complete the remaining study visits (96 remitted MDD patients and $48 \mathrm{HC}$ participants). Of these, 102 participants underwent resting-state $\mathrm{fMRI}$ scanning (63 remitted MDD patients and $39 \mathrm{HC}$ participants). group $(t(99)=3.71, p($ unc $)<0.0001)$. This was also true when comparing only melancholic rMDD patients with the HC group $(t(69)=4.06, p($ unc $)<0.0001)$. Despite this, the mean scores for both the rMDD patients (both overall and melancholic) were within the range of mild subthreshold depressive symptoms (Beck et al, 1988). Furthermore, the groups did not differ with respect to current MontgomeryAssberg Depression Rating Scale (MADRS) scores. The melancholic and nonmelancholic rMDD patients did not differ with respect to age, sex, number of MDEs, age of onset, months since remission, months since last psychotropic use, number of patients previously treated, number of suicide attempts, or family history of MDD. However, relative to the nonmelancholic rMDD group, melancholic patients had more years of education $(t(61)=2.61, p($ unc $)=0.01)$ and their last and most severe MDE was longer in duration $(t(61)=2.41, p($ unc $)=0.02)$ with MADRS scores indicating greater severity $(t(61)=3.28, p($ unc $)=0.002)$. These potential confounders were controlled for in subsequent analyses (see below).

\section{Image Acquisition}

Resting-state echo-planar images (EPIs) were acquired on a 3T Philips Achieva MRI scanner (Philips Medical Systems, Best, The Netherlands) with an 8-channel coil and were optimized for the detection of ventral frontal signal (240 volumes; 40 axial slices; $3 \mathrm{~mm}$ slice thickness; ascending sequential acquisition; repetition time: $2000 \mathrm{~ms}$; echo time: $22 \mathrm{~ms}$; field of view: $240 \times 240 \times 120 \mathrm{~mm}$; acquisition matrix: $80 \times 80$ voxels; reconstructed voxel size: $3 \mathrm{~mm}^{3}$; flip angle: $\left.90^{\circ}\right)$. Participants were instructed to lie still with eyes closed and to remain awake and were debriefed after scanning to ensure adherence to the instructions, at which time no participants reported having fallen asleep. A 3-dimensional T1-weighted magnetization-prepared rapid-acquisition gradient-echo (MPRAGE) structural image was also obtained for each participant (160 axial slices; $0.9 \mathrm{~mm}$ slice thickness; repetition time: $8.4 \mathrm{~ms}$; echo time: $3.9 \mathrm{~ms}$; field of view: $240 \times 191 \times 144 \mathrm{~mm}$; acquisition matrix: $256 \times 163$ voxels; reconstructed voxel size: $0.94 \times 0.94 \times 0.9 \mathrm{~mm}$; flip angle: $8^{\circ}$ ). For further clinical assessment, T2-weighted structural images were also acquired.

\section{Seed Region Selection}

The seed-based rsfMRI analyses were conducted using an a priori SCC region of interest (ROI; Montreal Neurological Institute (MNI) coordinates: $-4,23,-5 ; 6 \mathrm{~mm}$ sphere) that demonstrated functional decoupling during a task evoking feelings of guilt in patients vulnerable to experiencing MDEs (Green et al, 2012). The seed region used in the current study (MNI coordinates: $-4,23,-5$ ) is close in proximity to an SCC region identified in a separate study as demonstrating resting-state functional disconnection in patients vulnerable to MDD (MNI coordinates: 2, 23, - 6; Herringa et al, 2013). Taken together, the seed region used in this study was selected because of its association with depression vulnerability. Furthermore, although located more medially, the seed region's coordinates are close to SCC regions that demonstrated greater connectivity in current MDD patients 
Table 2 Demographic Variables in the Remitted MDD and HC Groups

\begin{tabular}{|c|c|c|c|c|c|c|c|c|}
\hline \multirow[t]{2}{*}{ Demographic Variables } & \multicolumn{2}{|c|}{$\mathrm{HC}(N=38)$} & \multicolumn{2}{|c|}{ rMDD $(N=63)$} & \multicolumn{2}{|c|}{$\begin{array}{c}\text { Melancholic rMDD } \\
\quad(N=33)\end{array}$} & \multicolumn{2}{|c|}{$\begin{array}{l}\text { Nonmelancholic } \\
\text { rMDD }(N=30)\end{array}$} \\
\hline & Mean & SD & Mean & SD & Mean & SD & Mean & SD \\
\hline Age & 36.2 & 13.8 & 36.4 & 12.3 & 37.7 & |l. | & 35 & 13.4 \\
\hline Years of education ${ }^{\mathrm{a}}$ & 16.8 & 2.3 & 16.8 & 2.4 & 17.5 & 2.2 & 16 & 2.4 \\
\hline Sex (male/female) & \multicolumn{2}{|c|}{$13 / 25$} & \multicolumn{2}{|c|}{$22 / 41$} & \multicolumn{2}{|c|}{$9 / 24$} & \multicolumn{2}{|c|}{$13 / 17$} \\
\hline Framewise displacement (mm) & 0.24 & 0.15 & 0.27 & 0.25 & 0.26 & 0.15 & 0.29 & 0.33 \\
\hline
\end{tabular}

Abbreviations: BDI, Beck Depression Inventory; HC, healthy control; MADRS, Montgomery-Åsberg Depression Rating Scale; MDD, major depressive disorder. With the exception of BDI scores, the remitted MDD patients and HC group did not significantly differ on the demographic variables (contingency coefficient <0.008, $p$ (unc) $>0.94 ; t<0.79, p$ (unc) $>0.43$ ). In addition, with the exception of BDI scores, the remitted melancholic MDD patients and $\mathrm{HC}$ group did not significantly differ on the demographic variables (contingency coefficient $<0.08, p(u n c)>0.52 ; t<1.30, p($ unc) $>0.20$ ). With the exception of years of education, the remitted melancholic and nonmelancholic MDD patients did not significantly differ on the demographic variables (contingency coefficient $<0.17, p($ unc) $>0.18 ; t<0.89, p($ unc) $>0.38$ ). a Significantly different between the remitted melancholic and nonmelancholic MDD groups $(t(6 \mathrm{l})=2.6 \mathrm{I}, p(\mathrm{unc})=0.0 \mathrm{I})$.

${ }^{b}$ Significantly different between the remitted MDD and HC groups $(t(99)=3.71, p($ unc $)<0.000$ I), and between the remitted melancholic MDD and HC groups $(t(69)=4.06, p($ unc $)<0.000$ I $)$

relative to HC participants (Greicius et al, 2007; Sheline et al, 2010).

\section{Resting-state fMRI Analysis}

EPIs and MPRAGE images were preprocessed using the Artifact Detection Tools (ART; http://web.mit.edu/swg/soft ware.htm), DPARSF Advanced Edition (Chao-Gan and YuFeng, 2010; http://rfmri.org/DPARSF), and SPM8 (http:// www.fil.ion.ucl.ac.uk/spm/) MATLAB (MathWorks) toolboxes. SPM8 was used for preprocessing to ensure compatibility with DPARSF. The first 10 volumes of the EPIs were discarded before slice timing and head motion correction. ART was used to produce regressors of high-motion volumes (ie, framewise signal intensity $>3 \mathrm{SD}$ from the global mean, framewise head displacement $>1 \mathrm{~mm}$ ). The MPRAGE images were coregistered to the EPIs and segmented. EPIs underwent linear detrending and nuisance covariates regression (24 motion parameters (Friston et al, 1996), white matter signal, cerebrospinal fluid signal, and ART regressors), normalization using nonlinear transformation parameters derived during segmentation, and smoothing using a $6 \mathrm{~mm}$ full-width at halfmaximum Gaussian kernel, before band-pass filtering to retain frequencies between 0.01 and $0.08 \mathrm{~Hz}$. Volume censoring was then performed to remove high-motion volumes identified by ART and resulting segments of uncensored data comprised of fewer than five contiguous volumes. All resulting EPIs contained $\geqslant 5$ min of data ( $\geqslant 150$ volumes).

Functional connectivity maps were computed using the resultant EPIs for each participant by correlating the average time course within the seed region with the time course of each voxel within the brain. The functional connectivity maps were then Fisher $Z$-transformed and entered into an analysis of variance (ANOVA) in SPM12 because it allows for cluster-level familywise error (FWE) correction of F-tests for differences between melancholic rMDD, nonmelancholic rMDD, and HC groups. Significance was determined using an uncorrected voxel-level cluster-forming threshold of $p<0.001$ and a cluster-level FWE-corrected threshold of $p<0.05$ across the whole-brain $(p<0.05)$ or a priori ROIs $(p<0.007$ corresponding to approximate Bonferronicorrected $p<0.05$ for the seven ROIs used in our analyses). The following bilateral a priori ROIs were defined: ventromedial prefrontal cortex, dorsomedial prefrontal cortex, anterior temporal cortex, amygdala, hippocampus, septal region, and hypothalamus. The definition of these ROIs has been described previously (Zahn et al, 2009) except for the hippocampal ROI that combined the left and right hippocampus masks from the Automatic Anatomical Labeling atlas (Tzourio-Mazoyer et al, 2002). These ROIs were chosen because of their close structural and/or functional connections with the SCC (Carmichael and Price, 1996; Johansen-Berg et al, 2008; Kondo et al, 2003; Vogt and Pandya, 1987), their role in the pathophysiology of depression (Elliott et al, 2011; Green et al, 2012; Mayberg, 2003), as well as in social cognitive and motivational processes (Elliott et al, 2011; Moll et al, 2005; Zahn et al, 2009). Mean Fisher $Z$-transformed correlation coefficients for each group were extracted from all clusters surviving FWE-correction and entered into a one-way ANOVA in SPSS 20 (SPSS, Chicago, IL) to probe pairwise post hoc comparisons between groups at Bonferroni-corrected $p<0.05$. The results of all statistical tests reported herein are two tailed.

\section{RESULTS}

A main effect of group was observed across the melancholic rMDD, nonmelancholic rMDD, and HC groups for connectivity between the SCC seed region and the left amygdala and right parahippocampal gyrus (Table 4 and Figure 1).

This was also reflected in significant effects for the extracted cluster averages in both regions (left amygdala: $\mathrm{F}(2,98)=11.2, p$ (unc) $<0.0001$; right parahippocampal gyrus: $\mathrm{F}(2,98)=13.5, p($ unc $)<0.0001)$. Bonferroni-corrected post hoc pairwise comparisons revealed decreased SCC connectivity 


\title{
Subgenual disconnection and melancholic depression
}

\author{
Cl Workman et al
}

Table 3 Clinical Characteristics of the Remitted Melancholic and Nonmelancholic MDD Patients

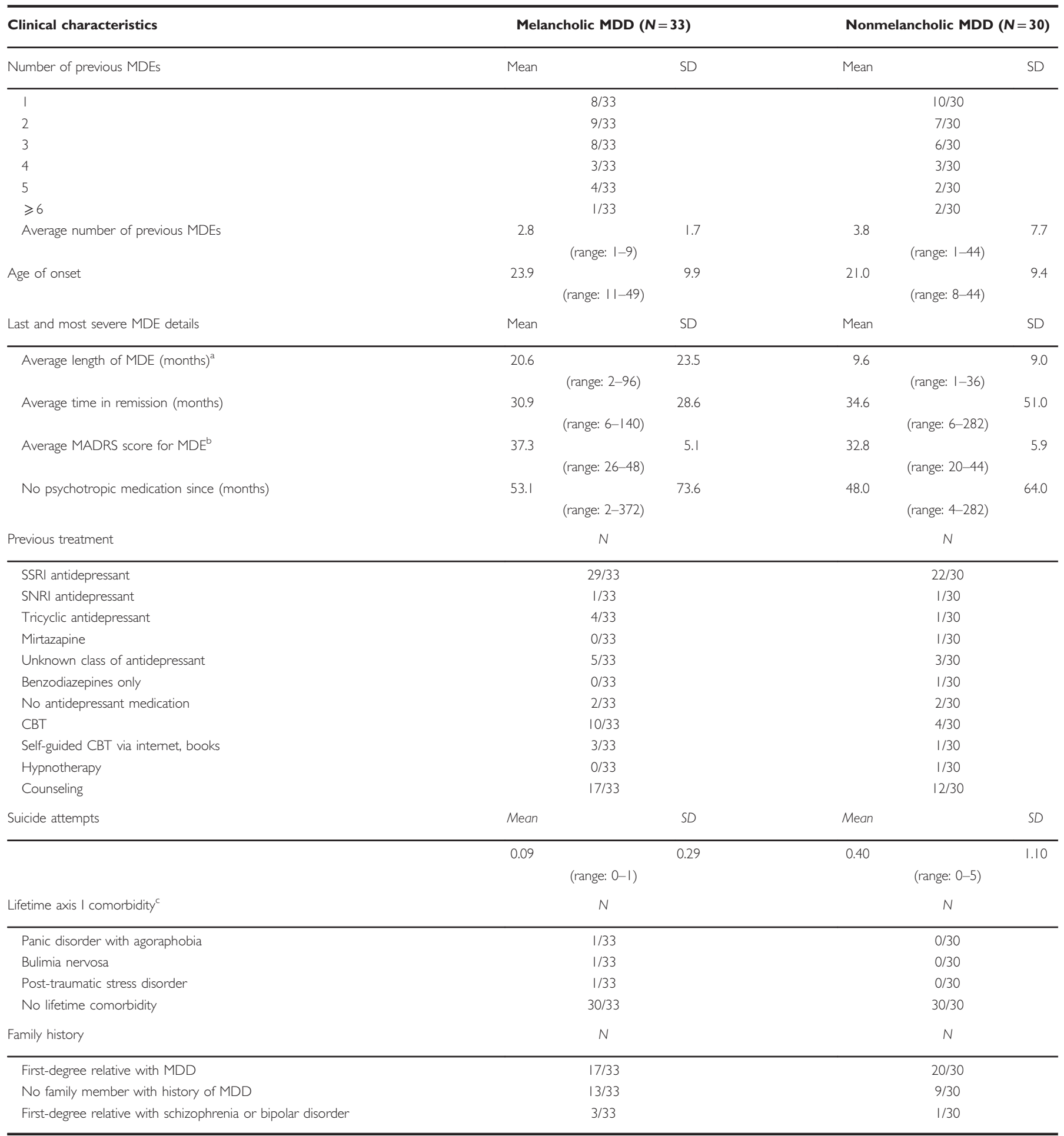

Abbreviations: CBT, cognitive behavioral therapy; MADRS, Montgomery-Åsberg Depression Rating Scale; MDD, major depressive disorder; MDE, major depressive episode; SSRI, selective serotonin reuptake inhibitor, SNRI, serotonin norepinephrine reuptake inhibitor.

All MDD participants stopped medication before the required washout phase. Means and SD are reported and/or the number of cases. Remitted melancholic and nonmelancholic MDD participants did not significantly differ on number of previous episodes, age of onset, average time in remission, average time since last taking psychotropic medications, number of patients previously treated, number of suicide attempts, lifetime axis I comorbidity, or family history (contingency coefficient < 0.21 , $p($ unc $)>0.09 ; t<1.55, p($ unc $)>0.13$ ). Furthermore, there were no differences between the remitted melancholic and nonmelancholic MDD participants regarding previous treatment with SSRIs, SNRIs, tricyclics, mirtazapine, or CBT (contingency coefficient $<0.21$, p(unc) $>0.10$ ).

${ }^{a}$ Significantly different between the remitted melancholic and nonmelancholic MDD groups $(t(6)=2.41, p($ unc $)=0.02)$.

bSignificantly different between the remitted melancholic and nonmelancholic MDD groups $(t(6 \mathrm{l})=3.28, p(\mathrm{unc})=0.002)$.

'All comorbid disorders were fully remitted at the time of study and none were likely to be the primary cause of the depressive episodes. 
Table 4 Resting-State Functional Disconnection in the Remitted Melancholic MDD Patients vs the Nonmelancholic Patients and HC Group

\begin{tabular}{|c|c|c|c|c|c|c|c|}
\hline \multirow[t]{2}{*}{ Hemisphere } & \multirow[t]{2}{*}{ Regions } & \multicolumn{3}{|c|}{ Peak MNI coordinates } & \multirow{2}{*}{$\begin{array}{c}\text { Peak } \\
\text { Z-score }\end{array}$} & \multirow{2}{*}{$\begin{array}{c}\text { Cluster } \\
\text { Size }\end{array}$} & \multirow{2}{*}{$\frac{\text { FWE corrected }}{\text { P-value }}$} \\
\hline & & $x$ & $Y$ & $\mathbf{Z}$ & & & \\
\hline \multicolumn{8}{|c|}{ FWE corrected at the cluster level over the whole brain $(p<0.05)$} \\
\hline $\mathrm{R}$ & Parahippocampal gyrus & 24 & -27 & -15 & 4.22 & 52 & 0.045 \\
\hline \multicolumn{8}{|c|}{ FWE corrected at the cluster level over an a priori amygdala $\mathrm{ROI}\left(p<0.007^{\mathrm{a}}\right)$} \\
\hline
\end{tabular}

Abbreviations: FWE, familywise error; HC, healthy control; L, left; MDD, major depressive disorder, MNI, Montreal Neurological Institute; R, right; ROI, region of interest. ${ }^{a}$ Corresponding to approximate Bonferroni-corrected $p<0.05$ /number of ROls to correct for the number of ROls used in the study.

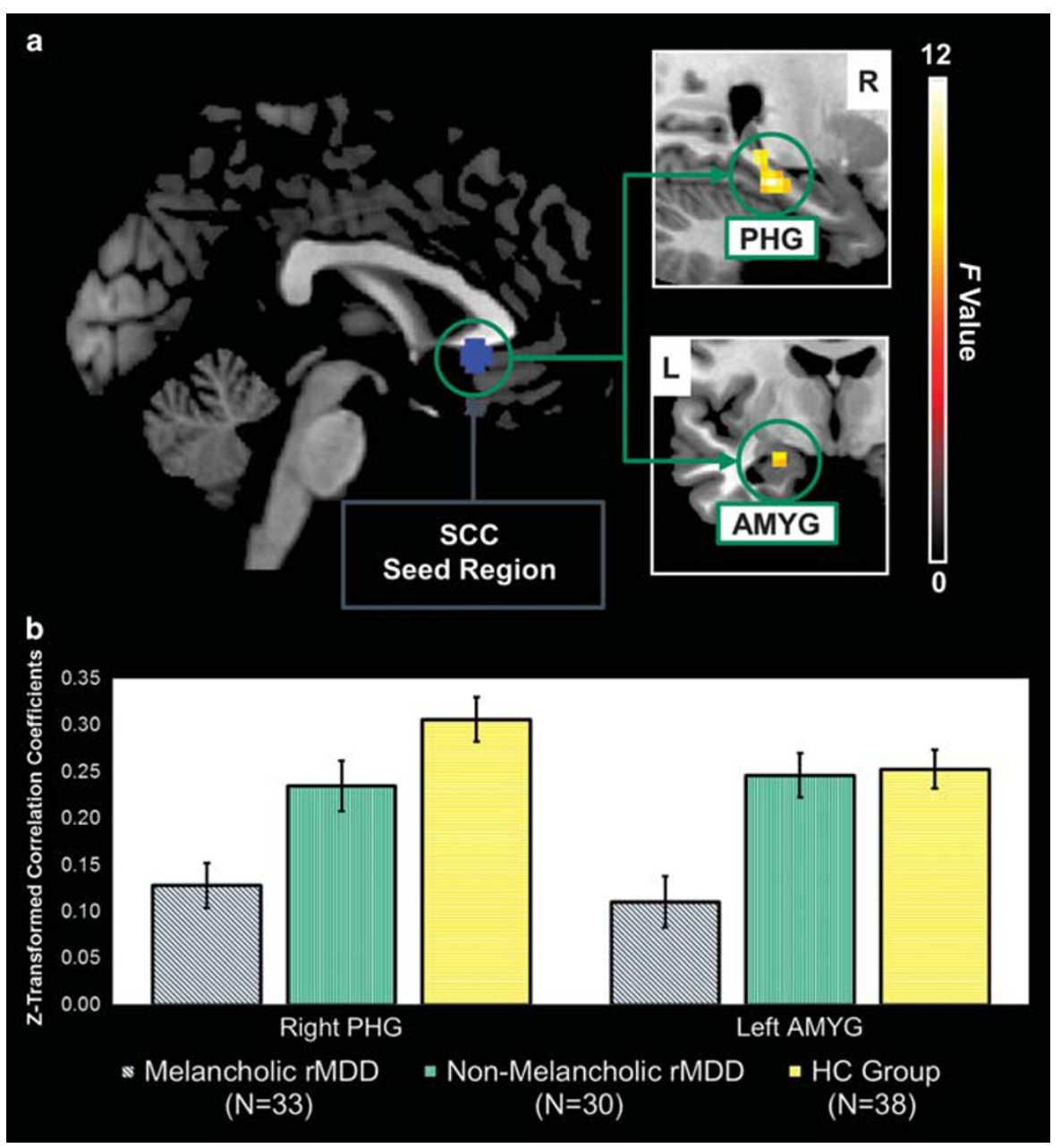

Figure I (a) The network of regions demonstrating resting-state functional disconnection with the subgenual cingulate seed region in the remitted melancholic MDD patients when compared with the remitted nonmelancholic MDD and HC groups. Whole-brain images were cropped and displayed at an uncorrected voxel-level threshold of $p<0.00 \mathrm{I}$. (b) Bar plots showing group differences in average Z-transformed correlation coefficients and SE for the right parahippocampal gyrus and left amygdala clusters. AMYG, amygdala; HC, healthy control; L, left; MDD, major depressive disorder; PHG, parahippocampal gyrus; R, right; SCC, subgenual cingulate cortex.

with the left amygdala in the melancholic rMDD patients $(\mathrm{M}=0.11, \mathrm{SD}=0.16)$ compared with both the nonmelancholic $\mathrm{rMDD}(\mathrm{M}=0.25, \mathrm{SD}=0.13, p=0.001$, mean difference $=$ $-0.14,95 \%$ CI $(-0.22,-0.05))$ and $\mathrm{HC}$ groups $(\mathrm{M}=0.25$, $\mathrm{SD}=0.13, p<0.0001$, mean difference $=-0.14,95 \% \mathrm{CI}(-0.22$, $-0.06)$ ). The same pattern of distinctively decreased SCC connectivity in melancholic rMDD emerged for the right parahippocampal gyrus $(\mathrm{M}=0.13, \mathrm{SD}=0.14)$ compared with the nonmelancholic $\operatorname{rMDD}(\mathrm{M}=0.23, \mathrm{SD}=0.15, p=0.013$, mean difference $=-0.11,95 \% \mathrm{CI}(-0.20,-0.02))$ and HC groups $(\mathrm{M}=0.31, \mathrm{SD}=0.15, p<0.0001$, mean difference $=$ $-0.18,95 \%$ CI $(-0.26,-0.09))$. 
SCC connectivity across the rMDD patients was not associated with the number of previous MDEs (left amygdala: $r_{\mathrm{s}}=-0.09, p$ (unc) $=0.50$; right parahippocampal gyrus: $\left.r_{\mathrm{s}}=-0.07, p(\mathrm{unc})=0.57\right)$. Across the melancholic and nonmelancholic rMDD groups, we found no evidence for effects of potentially confounding variables unrelated to subtype as SCC connectivity was neither associated with years of education (left amygdala: $r_{\mathrm{s}}=-0.005, p($ unc $)=0.97$; right parahippocampal gyrus: $r_{\mathrm{s}}=0.10, p(\mathrm{unc})=0.43$ ) nor current BDI scores (left amygdala: $r_{\mathrm{s}}=-0.08, p($ unc $)=0.55$; right parahippocampal gyrus: $r_{\mathrm{s}}=-0.06, p($ unc $\left.)=0.67\right)$. Of note, there were no group differences in mean framewise displacement, a measure of relative head displacement between contiguous volumes (Power et al, 2012) (Table 2).

In further analyses, we investigated whether categorical differences between rMDD subtypes in SCC connectivity could be dissociated from differences in past MDE severity and duration that are closely associated with the definition of melancholic vs nonmelancholic MDD. As expected, patients with longer past episode duration showed decreased SCC connectivity with both regions (left amygdala: $r_{\mathrm{s}}=-0.30$, $p$ (unc) $=0.02$; right parahippocampal gyrus: $r_{\mathrm{s}}=-0.32$, $p$ (unc $)=0.01)$ and there was a trend toward patients with more severe past episodes to show decreased SCC connectivity with the left amygdala $\left(r_{\mathrm{s}}=-0.23, p\right.$ (unc) $=$ 0.07). SCC connectivity was not, however, associated with past episode duration in the melancholic (left amygdala: $r_{\mathrm{s}}=-0.21, \quad p$ (unc $)=0.24$; right parahippocampal gyrus: $r_{\mathrm{s}}=-0.29, p(\mathrm{unc})=0.10$ ) or nonmelancholic (left amygdala: $r_{\mathrm{s}}=-0.10, p($ unc $)=0.61$; right parahippocampal gyrus: $r_{\mathrm{s}}=$ $-0.18, p($ unc $)=0.34)$ rMDD groups alone. There was a trend toward melancholic patients with more severe past MDEs to show increased connectivity with the right parahippocampal gyrus $\left(r_{\mathrm{s}}=0.33, p\right.$ (unc) $\left.=0.06\right)$ but not with the left amygdala $\left(r_{\mathrm{s}}=0.02, p(\right.$ unc $\left.)=0.92\right)$, and no such relationship was observed in the nonmelancholic patients (left amygdala: $r_{\mathrm{s}}=-0.27, p(\mathrm{unc})=0.15$; right parahippocampal gyrus: $r_{\mathrm{s}}=$ $-0.28, p($ unc $)=0.13)$. Taken together, this shows that a relationship between SCC connectivity and past episode duration may be a general feature of rMDD, but that this is not specific to either of the subtypes reported here. Additional exploratory analyses further showed that categorical differences in SCC connectivity between subtypes remained when adjusting for the effects of the duration (group difference adjusted for duration: left amygdala: $t(60)=2.93, p($ unc $)=0.005$; right parahippocampal gyrus: $t(60)=2.24, p($ unc $)=0.03$ ) and severity (group difference adjusted for severity, left amygdala: $t(60)=3.16, p($ unc $)=$ 0.002 ) of past episodes.

\section{DISCUSSION}

As predicted, patients vulnerable to melancholic MDEs demonstrated distinctive patterns of resting-state functional disconnection with the SCC when compared with nonmelancholic rMDD patients. This extends previous work showing that SCC metabolism is distinctively altered in current melancholic MDD (Pizzagalli et al, 2004). Intriguingly, contrary to our more specific predictions, we found SCC disconnection with the amygdala and parahippocampal gyrus rather than medial frontal cortices to be distinctive of melancholic rMDD. These group differences in functional connectivity were not because of potentially confounding effects of education, residual symptoms, or the duration and severity of past depressive episodes. Of note, the melancholic and nonmelancholic rMDD patients did not differ with respect to previous treatment for MDD, further strengthening the interpretation of group differences in SCC connectivity as correlates of subtype-specific vulnerability.

Our finding of SCC functional disconnection from the amygdala in melancholic rMDD is in agreement with reports of abnormal connectivity and activation of these structures in current MDD and MDD vulnerability. Lower functional connectivity between the SCC and amygdala was associated with vulnerability to experiencing depression in a healthy female cohort (Herringa et al, 2013). Kruschwitz et al (2014) recently demonstrated aberrant functional connectivity of the amygdala in individuals homozygous for the short allele of the 5-HTTLPR/rs25531 polymorphism, and the 5-HTTLPR polymorphism is known to confer depression risk. Interestingly, a support vector classification model incorporating resting-state connectivity of regions including the SCC and amygdala has shown promise for distinguishing patients with current MDD from a HC group (Craddock et al, 2009). Relatedly, SCC and amygdala reactivity to emotional stimuli was predictive of response to cognitive behavioral therapy in a cohort of MDD patients (Siegle et al, 2006). Patients with current MDD also exhibited microstructural white matter abnormalities between the SCC and amygdala (Cullen et al, 2010). Resting-state blood flow to the amygdala was associated with a negative emotional bias in rMDD patients following acute tryptophan depletion (Roiser et al, 2009). Amygdala activation in response to emotional faces has been widely reported as abnormal in current MDD (reviewed in Elliott et al, 2011) and its activation was associated with shame experiences in rMDD (Pulcu et al, 2014).

Our finding of SCC disconnection from the parahippocampal gyrus is in keeping with the known importance of medial temporal lobe structures in MDD. Situated in close proximity to both the amygdala and hippocampus, the parahippocampal gyrus is anatomically connected both to medial temporal structures (Amaral and Price, 1984) and the SCC (via the rostral cingulate; Vogt and Pandya, 1987). In agreement with our findings, lower resting-state SCChippocampal connectivity was described in a study of adolescents vulnerable to MDD (Herringa et al, 2013). Aberrant functional connectivity of the parahippocampal gyrus was also reported in rMDD patients during a sad mood induction paradigm (Zamoscik et al, 2014). Studies employing local measures of connectivity such as regional homogeneity consistently describe reductions in these measures in the parahippocampal gyrus in current MDD (reviewed in Dutta et al, 2014). In healthy volunteers, the parahippocampal gyrus has been shown to play a role in representing visual imagery (Downing et al, 2006) and in the retrieval of episodic, including autobiographical, memories (Gardini et al, 2006).

The network of decreased subgenual-amygdala-parahippocampal connectivity emerging from this study corresponds well to the limbic compartment of the limbic-cortical model of MDD put forth by Mayberg (2003). Mayberg (2003) suggests dysfunction within a limbic-cortical network 
is crucial in understanding the heterogeneity of MDD. In demonstrating disconnection within the limbic compartment associated with vulnerability to a specific MDD subtype, our findings support and extend this view. Notably, the limbic compartment in the model put forth by Mayberg (2003) is associated with somatic and vegetative symptoms that are commonly present in melancholic MDD.

Our finding that melancholic rMDD patients demonstrate decreased SCC connectivity runs counter to rsfMRI studies in current MDD reporting normalization with treatment of initially elevated SCC connectivity (Dutta et al, 2014; Greicius et al, 2007; Liston et al, 2014; Sheline et al, 2010). This discrepancy may reflect methodological differences across studies. For example, our seed region is located medially to the subgenual areas captured in rsfMRI studies in current MDD (Greicius et al, 2007; Sheline et al, 2010). This is an unlikely source of discrepancy, however, given that elevated resting-state activation of subgenual cingulate/septal areas is reported across studies in current MDD using a variety of imaging modalities and analysis techniques (reviewed in Drevets et al, 2008). A more probable explanation for the decreased SCC connectivity in the melancholic rMDD patients is that studies reporting normalization of SCC connectivity with treatment have been conducted in recently recovered patients instead of patients in full remission for several months as studied here (Liston et al, 2014). Indeed, the decreased connectivity we report in the melancholic rMDD patients is in keeping with a previous study in a cohort also vulnerable to depression that reported resting-state disconnection of the SCC (Herringa et al, 2013). Our results are also in keeping with a recent study that demonstrated decreased effective connectivity at rest in current melancholic MDD patients (Hyett et al, 2015). Furthermore, these results are in agreement with previous work showing guilt-selective decreases in functional connectivity in a rMDD group that included a large proportion of melancholic patients (Green et al, 2012). Given that MDD represents a lifetime diagnosis, this suggests abnormal SCC connectivity is a trait marker for melancholic MDD where the direction of connectivity is state dependent.

The following limitations of our study need to be discussed. It could be argued that use of a seed-based approach to analyze the rsfMRI data represents a limitation of the current study given the a priori assumptions required for seed selection (eg, see Dutta et al, 2014). However, SCC dysfunction in MDD is well established and using a SCC seed provides a direct link to the neuroimaging literature in MDD, including the study in rMDD from which the seed region was selected (Green et al, 2012). Another potential limitation of the current study is that we cannot firmly conclude whether decreased connectivity is associated with primary vulnerability that precedes the initial onset of MDD or secondary vulnerability where 'scarring' increases vulnerability to subsequent MDEs (Burcusa and Iacono, 2007). The lack of correlation between connectivity and number of previous MDEs, however, renders an association with primary vulnerability more likely. Another limitation to the current study is that we did not explicitly control for physiological artifacts associated with breathing and heart rate. Although such artifacts predominantly affect high frequencies not typically investigated with rsfMRI, changes to breathing or heart rate also appear as low-frequency fluctuations that may correlate with changes to BOLD signal, thereby spuriously increasing or creating patterns of functional connectivity (Murphy et al, 2013). We did, however, regress out white matter and cerebrospinal fluid signals that are thought to reflect such physiological artifacts (Dagli et al, 1999; Windischberger et al, 2002). Other strict controls for motion were also employed, such as regressing out an expanded set of 24 motion parameters (Friston et al, 1996; Power et al, 2014) and removing high-motion volumes (see, eg, Power et al, 2014) identified with the ART toolbox.

Taken together, the present study successfully used a SCC seed-based rsfMRI approach to identify patterns of decreased connectivity distinctive of melancholic rMDD. Melancholic rMDD patients demonstrated decreased connectivity of the SCC with the amygdala and parahippocampal gyrus when compared with the nonmelancholic rMDD and HC groups. These results provide the first resting-state neural signature distinctive for melancholic rMDD and may reflect a subtypespecific primary vulnerability factor for MDD. Longitudinal investigations of patients with a positive family history before their first MDE could be used to further validate these results as a biomarker of primary vulnerability to melancholic MDD.

\section{FUNDING AND DISCLOSURE}

JFWD has carried out consultancy and speaking engagements for Bristol Myers Squibb, AstraZeneca, Eli Lilly, Schering Plough, Janssen-Cilag, and Servier. All fees are paid to the University of Manchester as reimbursement for time taken. JFWD also has share options in P1Vital. RE has carried out consultancy for Cambridge Cognition. The other authors declare no conflict of interest.

\section{ACKNOWLEDGMENTS}

JM was supported by the LABS-D'Or Hospital Network, Rio de Janeiro, Brazil. JAG was funded by an Engineering and Physical Sciences Research Council (EPSRC) PhD studentship. RZ was funded by a Medical Research Council (MRC) Clinician Scientist Fellowship (G0902304).

\section{REFERENCES}

Amaral DG, Price JL (1984). Amygdalo-cortical projections in the monkey (Macaca fascicularis). J Comp Neurol 230: 465-496.

Beck AT, Steer RA, Brown GK (1996). Beck Depression Inventory-II. Psychological Corporation: San Antonio, TX.

Beck AT, Steer RA, Carbin MG (1988). Psychometric properties of the Beck Depression Inventory: twenty-five years of evaluation. Clin Psychol Rev 8: 77-100.

Burcusa SL, Iacono WG (2007). Risk for recurrence in depression. Clin Psychol Rev 27: 959-985.

Carmichael ST, Price JL (1996). Connectional networks within the orbital and medial prefrontal cortex of macaque monkeys. J Comp Neurol 371: 179-207.

Chao-Gan Y, Yu-Feng Z (2010). DPARSF: a MATLAB toolbox for 'pipeline' data analysis of resting-state fMRI. Front Syst Neurosc 4: 13.

Craddock RC, Holtzheimer PE, Hu XP, Mayberg HS (2009). Disease state prediction from resting state functional connectivity. Magn Reson Med 62: 1619-1628. 
Cullen KR, Klimes-Dougan B, Muetzel R, Mueller BA, Camchong J, Houri A et al (2010). Altered white matter microstructure in adolescents with major depression: a preliminary study. J Am Acad Child Adolesc Psychiatry 49: 173-183.e171.

Dagli MS, Ingeholm JE, Haxby JV (1999). Localization of cardiacinduced signal change in fMRI. Neuroimage 9: 407-415.

Downing PE, Chan AW, Peelen MV, Dodds CM, Kanwisher N (2006). Domain specificity in visual cortex. Cereb Cortex 16: 1453-1461.

Drevets WC, Savitz J, Trimble M (2008). The subgenual anterior cingulate cortex in mood disorders. CNS Spectrums 13: 663-681.

Dutta A, McKie S, Deakin JFW (2014). Resting state networks in major depressive disorder. Psychiatry Res 224: 139-151.

Ebert D, Martus P, Lungershausen E (1995). Change in symptomatology of melancholic depression over two decades. Psychopathology 28: 273-280.

Elliott R, Zahn R, Deakin JFW, Anderson IM (2011). Affective cognition and its disruption in mood disorders. Neuropsychopharmacology 36: 153-182.

First MB, Spitzer RL, Gibbon M, Williams JBW (2002). Structured Clinical Interview for DSM-IV-TR Axis I Disorders, Research Version, Patient Edition. (SCID-I/P). Biometrics Research, New York State Psychiatric Institute: New York, NY.

Fox MD, Raichle ME (2007). Spontaneous fluctuations in brain activity observed with functional magnetic resonance imaging. Nat Rev Neurosci 8: 700-711.

Friston KJ, Williams S, Howard R, Frackowiak RSJ, Turner R (1996). Movement-related effects in fMRI time-series. Magn Reson Med 35: 346-355.

Gaffrey MS, Luby JL, Botteron K, Repovš G, Barch DM (2012). Default mode network connectivity in children with a history of preschool onset depression. J Child Psychol Psychiatry 53: 964-972.

Gardini S, Cornoldi C, De Beni R, Venneri A (2006). Left mediotemporal structures mediate the retrieval of episodic autobiographical mental images. Neuroimage 30: 645-655.

Green S, Lambon Ralph MA, Moll J, Deakin JFW, Zahn R (2012). Guilt-selective functional disconnection of anterior temporal and subgenual cortices in major depressive disorder. Arch Gen Psychiatry 69: 1014-1021.

Greicius MD, Flores BH, Menon V, Glover GH, Solvason HB, Kenna H et al (2007). Resting-state functional connectivity in major depression: abnormally increased contributions from subgenual cingulate cortex and thalamus. Biol Psychiatry 62: 429-437.

Hecht H, van Calker D, Berger M, von Zerssen D (1998). Personality in patients with affective disorders and their relatives. J Affect Disord 51: 33-43.

Herringa RJ, Birn RM, Ruttle PL, Burghy CA, Stodola DE, Davidson RJ et al (2013). Childhood maltreatment is associated with altered fear circuitry and increased internalizing symptoms by late adolescence. Proc Natl Acad Sci USA 110: 19119-19124.

Hyett MP, Breakspear MJ, Friston KJ, Guo CC, Parker GB (2015). Disrupted effective connectivity of cortical systems supporting attention and interoception in melancholia. JAMA Psychiatry 72: 350-358.

Johansen-Berg H, Gutman DA, Behrens TEJ, Matthews PM, Rushworth MFS, Katz E et al (2008). Anatomical connectivity of the subgenual cingulate region targeted with deep brain stimulation for treatment-resistant depression. Cereb Cortex 18: 1374-1383.

Kondo H, Saleem KS, Price JL (2003). Differential connections of the temporal pole with the orbital and medial prefrontal networks in macaque monkeys. J Comp Neurol 465: 499-523.

Kruschwitz JD, Walter M, Varikuti D, Jensen J, Plichta MM, Haddad L et al (2014). 5-HTTLPR/rs25531 polymorphism and neuroticism are linked by resting state functional connectivity of amygdala and fusiform gyrus. Brain Struct Funct 220: 2373-2385.

Kupfer DJ (1991). Long-term treatment of depression. J Clin Psychiatry 52(Suppl): 28-34.

Liston C, Chen AC, Zebley BD, Drysdale AT, Gordon R, Leuchter B et al (2014). Default mode network mechanisms of transcranial magnetic stimulation in depression. Biol Psychiatry 76: 517-526.

Mayberg HS (2003). Modulating dysfunctional limbic-cortical circuits in depression: towards development of brain-based algorithms for diagnosis and optimised treatment. Br Med Bull 65: 193-207.

Meyer-Lindenberg A, Weinberger DR (2006). Intermediate phenotypes and genetic mechanisms of psychiatric disorders. Nat Rev Neurosci 7: 818-827.

Moll J, Zahn R, de Oliveira-Souza R, Krueger F, Grafman J (2005). Opinion: the neural basis of human moral cognition. Nat Rev Neurosci 6: 799-809.

Murphy K, Birn RM, Bandettini PA (2013). Resting-state fMRI confounds and cleanup. Neuroimage 80: 349-359.

Pizzagalli DA, Oakes TR, Fox AS, Chung MK, Larson CL, Abercrombie HC et al (2004). Functional but not structural subgenual prefrontal cortex abnormalities in melancholia. Mol Psychiatry 9: 393-405.

Power JD, Barnes KA, Snyder AZ, Schlaggar BL, Petersen SE (2012). Spurious but systematic correlations in functional connectivity MRI networks arise from subject motion. Neuroimage 59: 2142-2154.

Power JD, Mitra A, Laumann TO, Snyder AZ, Schlaggar BL, Petersen SE (2014). Methods to detect, characterize, and remove motion artifact in resting state fMRI. Neuroimage 84: 320-341.

Pulcu E, Lythe KE, Elliott R, Green S, Moll J, Deakin JFW et al (2014). Increased amygdala response to shame in remitted major depressive disorder. PLoS One 9: e86900.

Ressler KJ, Mayberg HS (2007). Targeting abnormal neural circuits in mood and anxiety disorders: from the laboratory to the clinic. Nat Neurosci 10: 1116-1124.

Roiser JP, Levy J, Fromm SJ, Nugent AC, Talagala SL, Hasler G et al (2009). The effects of tryptophan depletion on neural responses to emotional words in remitted depression. Biol Psychiatry 66: 441-450.

Sheline YI, Price JL, Yan Z, Mintun MA (2010). Resting-state functional MRI in depression unmasks increased connectivity between networks via the dorsal nexus. Proc Natl Acad Sci USA 107: 11020-11025.

Siegle GJ, Carter CS, Thase ME (2006). Use of FMRI to predict recovery from unipolar depression with cognitive behavior therapy. Am J Psychiatry 163: 735-738.

Tzourio-Mazoyer N, Landeau B, Papathanassiou D, Crivello F, Etard O, Delcroix N et al (2002). Automated anatomical labeling of activations in SPM using a macroscopic anatomical parcellation of the MNI MRI single-subject brain. Neuroimage 15: 273-289.

Vogt BA, Pandya DN (1987). Cingulate cortex of the rhesus monkey: II. Cortical afferents. J Comp Neurol 262: 271-289.

Windischberger $\mathrm{C}$, Langenberger $\mathrm{H}$, Sycha $\mathrm{T}$, Tschernko EM, Fuchsjäger-Mayerl G, Schmetterer L et al (2002). On the origin of respiratory artifacts in BOLD-EPI of the human brain. Magn Reson Imaging 20: 575-582.

Zahn R, Lythe KE, Gethin JA, Green S, Deakin JFW, Workman CI et al (2015). Negative emotions towards others are diminished in remitted major depression. Eur Psychiatry 30: 448-453.

Zahn R, Moll J, Paiva M, Garrido G, Krueger F, Huey ED et al (2009). The neural basis of human social values: evidence from functional MRI. Cereb Cortex 19: 276-283.

Zamoscik V, Huffziger S, Ebner-Priemer U, Kuehner C, Kirsch P (2014). Increased involvement of the parahippocampal gyri in a sad mood predicts future depressive symptoms. Soc Cogn Affect Neurosci 9: 2034-2040. 